\title{
Viscoelastic flow in a 3D square/square contraction: Visualizations and simulations
}

\author{
M. A. Alves ${ }^{\text {a) }}$ \\ Faculdade de Engenharia da Universidade do Porto, Departamento de \\ Engenharia Química, CEFT, Rua Dr. Roberto Frias, 4200-465 Porto, Portugal
}

F. T. Pinho ${ }^{b)}$

Centro de Estudos de Fenómenos de Transporte, Faculdade de Engenharia da Universidade do Porto, 4200-465 Porto, Portugal and Universidade do Minho, Largo do Paço, 4704-553 Braga, Portugal

P. J. Oliveira ${ }^{\text {) }}$

Departamento de Engenharia Electromecânica, Unidade de Materiais Têxteis e Papeleiros, Universidade da Beira Interior, 6201-001 Covilhã, Portugal

(Received 1 December 2007; final revision received 26 August 2008)

\section{Synopsis}

The inertialess three-dimensional (3D) flow of viscoelastic shear-thinning fluids in a 4:1 sudden square-square contraction was investigated experimentally and numerically and compared with the flow of inelastic fluids. Whereas for a Newtonian fluid the vortex length remains unchanged at low Reynolds numbers, with the non-Newtonian fluid there is a large increase in vortex length with fluid elasticity leading to unstable periodic flow at higher flow rates. In the steady flow regime the vortices are 3D and fluid particles enter the vortex at the middle plane, rotate towards its eye, drift sideways to the corner-plane vortex, rotate to its periphery, and exit to the downstream duct. Such dynamic process is reverse of that observed and predicted with Newtonian fluids. Numerical predictions using a multimode Phan-Thien-Tanner viscoelastic model are found to match the visualizations accurately and in particular are able to replicate the observed flow reversal. The effect of fluid rheology on flow reversal, vortex enhancement, and entry pressure drop is investigated in detail. (C) 2008 The Society of Rheology. [DOI: 10.1122/1.2982514]

\section{INTRODUCTION}

Matching experiments and simulations in non-Newtonian flow systems is a matter of great relevance as illustrated in the recent study of Collis et al. (2005). It is useful to assess the adequacy of constitutive models and it may serve to explain or highlight new

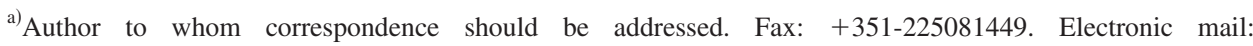
mmalves@fe.up.pt

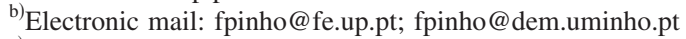

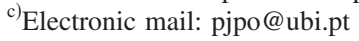


fluid mechanics phenomena. In order to achieve these goals, it is necessary to select simple but useful flow geometries.

Sudden contraction flows are classical benchmark problems used in computational rheology [Hassager (1988); Brown and McKinley (1994)] and possess features found in many industrial situations, such as in extrusion processes. Consequently, a large number of experimental and numerical investigations can be found in the literature, although most are concerned with nominally two-dimensional (2D) planar and axisymmetric contractions [e.g., Cable and Boger (1978a, 1978b, 1979); Walters and Rawlinson (1982); Crochet et al. (1984); Evans and Walters (1986, 1988); Boger et al. (1986); Boger (1987); McKinley et al. (1991); Coates et al. (1992); Boger and Walters (1993); Quinzani et al. (1994); Rothstein and McKinley (1999); Lee et al. (2001); Owens and Phillips (2002); Walters and Webster (2003); Alves et al. (2004); Collis et al. (2005); Oliveira et al. (2007)]. In spite of the geometrical simplicity, the flow behavior of non-Newtonian fluids in contraction flows can be very surprising, and different flow patterns are observed even for fluids with similar rheological behavior [Boger et al. (1986); Owens and Phillips (2002)].

Studies related to three-dimensional (3D) sudden contractions are less common, in spite of the practical relevance of this geometry, and the amount of published research on this topic has been scarce. The present work presents new experimental results based on flow visualizations, complemented with supporting results from numerical simulations, for Newtonian and viscoelastic shear-thinning fluids in a 4:1 sudden square/square (SQ/ SQ) contraction. In a previous investigation [Alves et al. (2005)], the behavior of Boger fluids in the same geometry was visualized and a review of past work on the 2D planar and axisymmetric geometries was presented. Therefore, in this section we concentrate mainly on the $3 \mathrm{D}$ case.

To our knowledge, the first work on viscoelastic flows in 3D square contractions was published by Walters and Webster (1982) who found similarities with the flow through a circular contraction. Walters and Rawlinson (1982) also confirmed that the differences between the flows in planar and circular contractions also existed when comparing the flows in planar and the 13.3:1 SQ/SQ contractions. The experiments and numerical calculations of Purnode and Crochet (1996) confirmed the similarities between the main flow features in 2D and 3D flows, and they concluded that lip vortices should not be associated with inertial effects. However, these authors also found that full capture of 3D effects required 3D computations and especially an accurate representation of fluid rheology.

As a precursor to the present contribution, Alves et al. (2005) carried out visualizations with polyacrylamide-based Boger fluids in a 4:1 SQ/SQ geometry and found a nonmonotonic variation of the recirculation length with the flow rate. The corner vortex was found to increase initially (for one of the fluids), peaking at a Deborah number ${ }^{1}$ of around 6 , followed by a strong decrease of length to a minimum at $\mathrm{De}_{2} \approx 15-20$, for both fluids analyzed. Then, as the Deborah number increased further the vortex length increased significantly until an unstable periodic flow was established at $\mathrm{De}_{2} \approx 45$ for the more concentrated Boger fluid and at $\mathrm{De}_{2} \approx 52$ for the other. This increase in vortex length was preceded by a divergence pattern of the streamlines, a typical behavior of Boger fluids related to their extensional properties, as discussed by Alves and Poole (2007).

\footnotetext{
${ }^{1} \mathrm{De}_{2}=\lambda U_{2} / H_{2}$, where $U_{2}$ and $H_{2}$ are the downstream channel bulk velocity and half side, respectively, and $\lambda$ is the relaxation time of the fluid.
} 
Predictions of 3D contraction flows are scarce due to the large computational demands involved, but are in much need due to their relevance in practical situations. Mompean and Deville (1997) used a finite-volume methodology in a staggered grid to investigate the flow in a quasi-3D planar contraction, one that tends to become $2 \mathrm{D}$ when the aspect ratio is large enough, and compared qualitatively their results with the measurements of Quinzani et al. (1994) for a 4:1 2D planar contraction.

The 3D simulations of Xue et al. (1998a) for upper-convected Maxwell (UCM) and Phan-Thien-Tanner (PTT) fluids also employed a finite-volume method applied to a quasiplanar 4:1 contraction flow. For the corresponding 3D SQ/SQ contraction, Xue et al. (1998b) carried out a set of simulations with the same finite-volume method, using a PTT model having nonzero second normal stress differences and UCM constitutive equations. Vortex enhancement for both the constant viscosity and the shear-thinning viscoelastic fluids was found for both cases. In contrast, for the 2D 4:1 planar contraction only the PTT fluid exhibited vortex enhancement. These differences replicate those between axisymmetric and planar contractions and result from the equivalent Hencky strains generated in the circular and the SQ/SQ cases. This investigation concentrated on relating the flow dynamics with the transient extensional viscosity behavior of the fluids, but nothing was mentioned regarding secondary flows in the contraction region or the onset of flow instabilities. Xue et al. (1998b) reported secondary flows in the fully developed upstream and downstream square duct flows for the case of PTT fluid, but these were exclusively due to nonzero second normal stress differences.

More recently, Sirakov et al. (2005) studied the flow through 3D contractions using the eXtended Pom-Pom model; the geometry differed from the present one in that the exiting die was not a square duct but presented either a rectangular or a circular cross section. They found that the recirculation was an open vortex in which the fluid followed a complicated spiraling motion. More interestingly, for the square/circular contraction case, they noticed that the flow direction in the recirculation was reversed in the case of the viscoelastic fluid, as compared to the Newtonian case, a result which confirmed the previous observations of Alves et al. (2003c) for a SQ/SQ contraction.

It is clear that much remains to be known for the $3 \mathrm{D} S \mathrm{SQ} / \mathrm{SQ}$ contraction and the increase in computational power over the last years has now made it possible to accurately perform numerical simulations of true $3 \mathrm{D}$ flows in reasonable time. This is the motivation for our work on SQ/SQ contractions and the present experimental and numerical work complements the previous experimental investigation of Alves et al. (2005) who considered Boger fluids only. Here, visualizations and numerical predictions of the flow of a viscoelastic shear-thinning fluid in a 4:1 SQ/SQ contraction were carried out in much greater detail.

In the next section the experimental apparatus is briefly described and the results of the rheological measurements of the fluids are discussed. The numerical method and the constitutive equations used in the numerical solution are then briefly outlined in Sec. III and finally, in Sec. IV, results of the experiments and numerical simulations are presented and discussed in detail. The paper ends with the main conclusions of this work.

\section{EXPERIMENTAL CONDITIONS}

\section{A. Experimental rig}

The experimental rig, schematically shown in Fig. 1, was assembled as described in Alves et al. (2005), and the experiments were performed in a dark room. A $1000 \mathrm{~mm}$ long square duct having sides of $2 H_{1}=24.0 \mathrm{~mm}$ was followed by a second $300 \mathrm{~mm}$ long square duct of sides $2 \mathrm{H}_{2}=6.0 \mathrm{~mm}$ defining the $3 \mathrm{D} 4: 1$ side contraction ratio (CR 


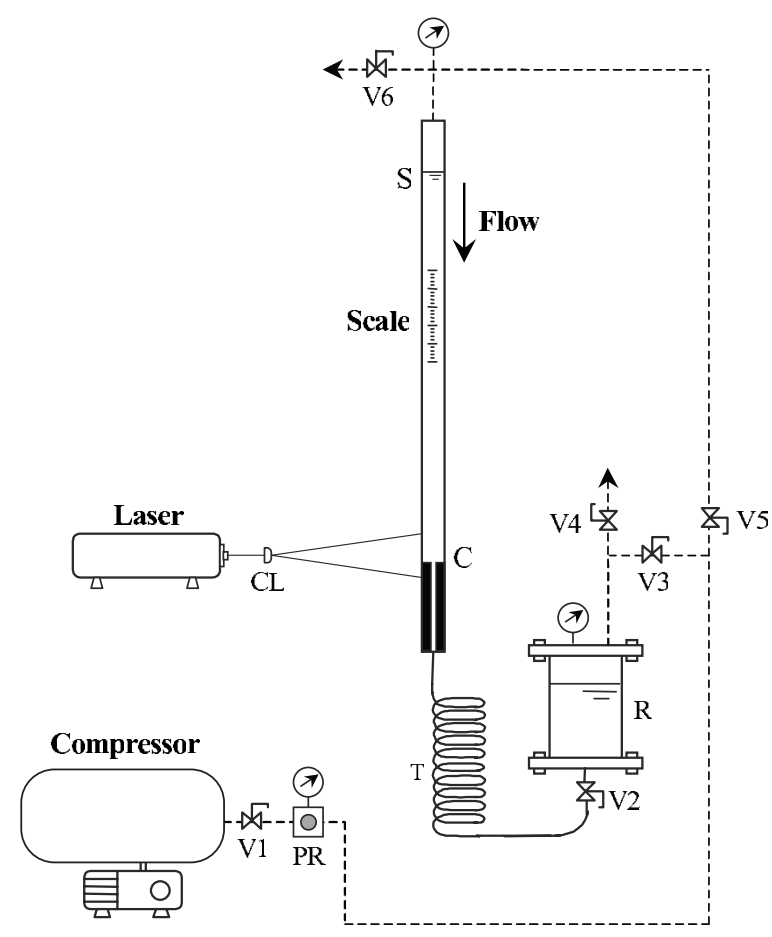

FIG. 1. Schematic representation of the experimental setup: $\mathrm{C}-$ contraction; $\mathrm{CL}-$ cylindrical lens; PRpressure regulator; R-reservoir; S-Liquid surface; T-long coiled tube; V1-V6- ball valves.

$\left.=H_{1} / H_{2}\right)$. The flow rate was set by an adequate control of applied pressure on the upstream duct (dashed lines represent pressurized air lines, with applied pressures between 0.5 and 4 bar) and frictional losses in the long coiled tube (T) located at the bottom of the rig. The flow rate was measured by the stop-watch and level marker technique and the fluid temperature was monitored to properly take into account the fluid properties.

The flow was illuminated with a light sheet generated from a $10 \mathrm{~mW} H \mathrm{He}-\mathrm{Ne}$ laser light source after the beam passed a cylindrical lens to create a sheet of light which illuminated highly reflective tracer particles suspended in the fluid $(10 \mu \mathrm{m}$ PVC particles). Their trajectories were recorded using long time exposure photography with a CANON EOS300 camera using an EF100 mm f/2.8 macrolens. For the smaller velocities very long exposure times were necessary (above $1 \mathrm{~h}$ ) and Hoya neutral-density filters were used (ranging from $2 \times$ to $400 \times$ light transmittance reduction).

\section{B. Rheological characterization of the fluids}

The rheological properties of the viscoelastic fluids were measured by an AR2000 rheometer from TA Instruments with cone-plate geometry $\left(40 \mathrm{~mm}\right.$ diameter and $2^{\circ}$ angle). The shear viscosity $(\eta)$ and the first normal stress difference coefficient $\left(\Psi_{1}\right)$ were measured in steady shear flow, and the storage and loss moduli $\left(G^{\prime}, G^{\prime \prime}\right)$ in dynamic shear flow. To measure the viscosity of the Newtonian fluids a falling ball viscometer from Gilmont Instruments (ref. GV-2200) was also used.

Three fluids, listed in Table I, were investigated in this study: two viscous Newtonian fluids (N85 and N91) and a moderately shear-thinning viscoelastic fluid (PAA500) made with polyacrylamide (Separan AP30 from SNF Floerger). The non-Newtonian fluid was 
TABLE I. Composition of fluids in mass concentrations and density.

\begin{tabular}{ccccccc}
\hline \hline Designation & PAA (ppm) & Glycerin $(\%)$ & Water $(\%)$ & $\mathrm{NaCl}(\%)$ & Kathon $(\mathrm{ppm})$ & $\rho\left(\mathrm{kg} / \mathrm{m}^{3}\right)^{\mathrm{a}}$ \\
\hline N85 & - & 84.99 & 15.01 & - & 25 & 1221 \\
N91 & - & 90.99 & 7.51 & 1.50 & 25 & 1250 \\
PAA500 & 500 & 84.97 & 14.98 & - & 25 & 1226 \\
\hline \hline
\end{tabular}

${ }^{\mathrm{a}}$ Measured at 294.4 K.

prepared by dissolving the required amount of PAA onto the Newtonian solvent of moderate viscosity (N85). To avoid bacteriological degradation the biocide Kathon LXE from Rohm and Haas was also added to all fluids at a concentration of $25 \mathrm{ppm}$. The fluid densities were measured at $294.4 \mathrm{~K}$ with a picnometer and are included in Table I.

For the N85 fluid the shear viscosity was $\eta=0.125 \mathrm{~Pa} \mathrm{~s}$ at $291.2 \mathrm{~K}$, the temperature at which the visualizations with this fluid took place, whereas for the N91 fluid the rheometer was used to measure the shear viscosity in the range of temperatures from 289.1 to $298.2 \mathrm{~K}$. As described in Alves et al. (2005), the effect of temperature on the viscosity was accounted for by the Arrhenius equation

$$
\ln \left(a_{T}\right)=\ln \left(\frac{\eta}{\eta_{0}}\right)=\left[\frac{\Delta H}{R}\left(\frac{1}{T}-\frac{1}{T_{0}}\right)\right],
$$

where $T$ is the absolute temperature and $T_{0}$ is the reference absolute temperature. For the N91 fluid, fitting of this equation to the experimental viscosity $(\eta)$ data gave $\Delta H / R$ $=6860 \mathrm{~K}$ and $\eta_{0}=0.367 \mathrm{Pas}$ at $T_{0}=293.2 \mathrm{~K}$. More extensive measurements with fluid N91 indicated a systematic uncertainty in the measurement of $N_{1}$ of $\pm 10 \mathrm{~Pa}$ and the onset of inertial effects for $\dot{\gamma}>100 \mathrm{~s}^{-1}$ which was well predicted by theory [Alves et al. (2005)].

For the PAA500 solution, the dynamic measurements of the storage and loss moduli taken at $288.2 \mathrm{~K}$ allowed the determination of a linear viscoelastic spectrum. These are listed in Table II and the corresponding dynamic viscosity, $\eta^{\prime}$, and $2 G^{\prime} / \omega^{2}$ predictions (lines) are compared with the experiments (symbols) in Figs. 2 and 3, respectively. We note that the solvent viscosity used in the fitting is somehow higher than the real shear viscosity of the solvent at the reference temperature. If we chose to match the Newtonian component of the shear viscosity to that of the base solvent we needed to use at least one more mode in the PTT model, otherwise predictions of $G^{\prime}$ and $G^{\prime \prime}$ would not be as good. We chose to use a maximum of four modes (plus solvent) to avoid using too many adjustable parameters and determined the Newtonian solvent viscosity (as the other parameters) from a least square fitting so that the prediction of the rheometric data was as

TABLE II. Linear viscoelastic spectra for the PAA500 fluid at $T_{0}$ $=288.2 \mathrm{~K}$.

\begin{tabular}{ccc}
\hline \hline Mode $k$ & $\lambda_{k} / \mathrm{s}$ & $\eta_{k} / \mathrm{Pas}$ \\
\hline 1 & 30 & 2.5 \\
2 & 3 & 0.9 \\
3 & 0.3 & 0.3 \\
4 & 0.03 & 0.1 \\
Solvent & - & 0.27 \\
\hline \hline
\end{tabular}




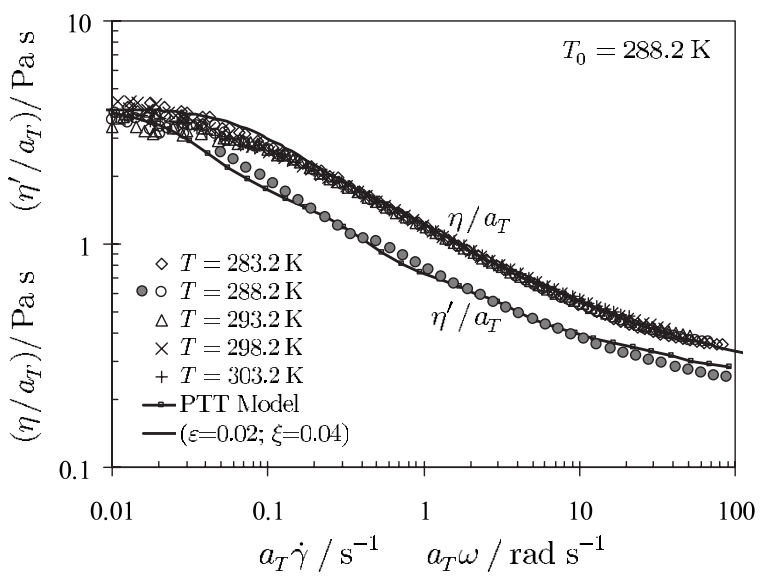

FIG. 2. Measured (symbols) normalized shear viscosity $(\eta)$ and dynamic viscosity $\left(\eta^{\prime}\right)$ of the PAA500 fluid at different temperatures and fit (lines) with the four-mode linear PTT model.

good as possible. Nevertheless, it is clear that, within the measured frequency range, a four-mode model is adequate to represent accurately the dynamic rheology of the fluid.

The steady shear tests were carried out for temperatures between 283.2 and $303.2 \mathrm{~K}$ from which the shift factor $a_{T}$ was determined. Using as reference the temperature of 288.2 K, the temperature at which the visualizations with PAA500 fluid took place, the ratio $\Delta H / R=5900 \mathrm{~K}$ was obtained. The reduced shear viscosity $\left(\eta / a_{T}\right.$ versus $\left.a_{T} \dot{\gamma}\right)$ for this fluid is represented in Fig. 2. The PAA500 solution is only moderately shear thinning because its viscosity decreases only by a factor of 15 between the low and high shear-rate values.

To model the rheological behavior of the PAA500 fluid, a four-mode linear PTT model plus solvent was selected (see Sec. III for the formalism) and its prediction for the shear viscosity is plotted also as a full line in Fig. 2. Besides the parameters listed in Table II, and based on the low amplitude dynamic shear behavior, the PTT model contains two extra parameters $(\varepsilon=0.02$ and $\xi=0.04)$, which were determined from fitting to the master

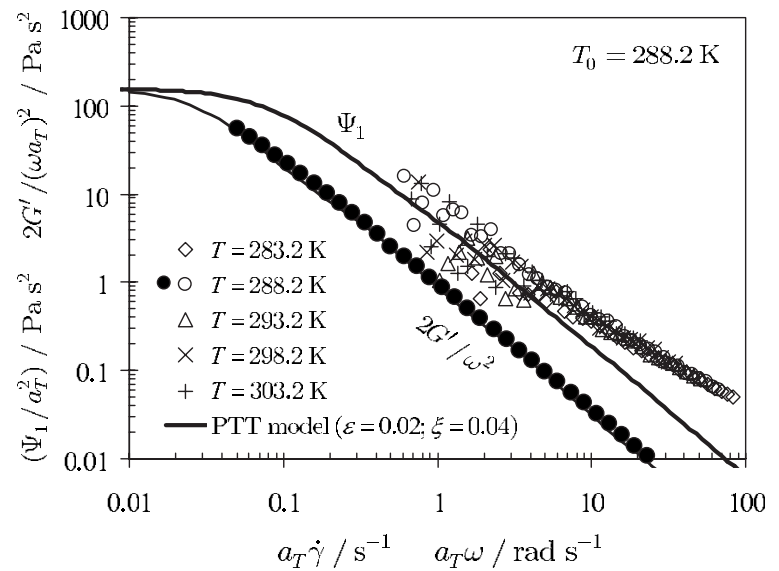

FIG. 3. Comparison between measured (symbols) master curves of $\Psi_{1} / a_{T}^{2}$ and $2 G^{\prime} /\left(\omega a_{T}\right)^{2}$ for the PAA500 fluid with the predictions (lines) by the fitted four-mode linear PTT model. 
curves of the shear viscosity and the first normal-stress difference coefficient $\left(\Psi_{1} / a_{T}^{2}\right)$. As can be seen, the linear PTT adequately adjusts the shear viscosity, but predicts a more intense shear-thinning in $\Psi_{1}$ than the rheological experiments. These latter data sets can be seen in Fig. 3 where the experimental results for $\Psi_{1} / a_{T}^{2}$ versus $a_{T} \dot{\gamma}$ and for $2 G^{\prime} /\left(\omega a_{T}\right)^{2}$ versus $a_{T} \omega$ are compared with predictions by the fitted model.

\section{OVERVIEW OF THE NUMERICAL SIMULATION METHOD}

\section{A. Brief description of the numerical method}

The equations that describe incompressible viscoelastic fluid flow are the equations of conservation of mass

$$
\boldsymbol{\nabla} \cdot \mathbf{u}=0
$$

linear momentum

$$
\rho\left[\frac{\partial \mathbf{u}}{\partial t}+\mathbf{u} \cdot \boldsymbol{\nabla} \mathbf{u}\right]=-\nabla p+\nabla \cdot \tau_{p}+\eta_{s} \nabla^{2} \mathbf{u},
$$

and a constitutive equation for the polymeric contribution to the extra stress, $\boldsymbol{\tau}_{p}$. In these equations $\mathbf{u}$ represents the velocity vector, $p$ is the pressure, and $\rho$ is the density. The extra stress is given by the sum of a Newtonian solvent contribution of viscosity $\eta_{s}$ and a polymer contribution $\tau_{p}$ which introduces viscoelasticity.

The polymer contribution $\tau_{p}$ is expressed as a sum of $N$ viscoelastic modes $(k)$,

$$
\boldsymbol{\tau}_{p}=\sum_{k=1}^{N} \boldsymbol{\tau}_{p, k}
$$

each of which obeys an equation of the form

$$
\begin{aligned}
Y\left[\operatorname{tr}\left(\boldsymbol{\tau}_{p, k}\right)\right] \boldsymbol{\tau}_{p, k}+\lambda_{k}\left[\frac{\partial \boldsymbol{\tau}_{p, k}}{\partial t}+\mathbf{u} \cdot \boldsymbol{\nabla} \boldsymbol{\tau}_{p, k}\right]= & 2 \eta_{p, k} \mathbf{D}+\lambda_{k}\left[\boldsymbol{\tau}_{p, k} \cdot \boldsymbol{\nabla} \mathbf{u}+\boldsymbol{\nabla} \mathbf{u}^{T} \cdot \boldsymbol{\tau}_{p, k}\right. \\
& \left.-\xi\left(\boldsymbol{\tau}_{p, k} \cdot \mathbf{D}+\mathbf{D} \cdot \boldsymbol{\tau}_{p, k}\right)\right] .
\end{aligned}
$$

More specifically, a four mode PTT model with a linear stress coefficient [Phan-Thien and Tanner (1977)] was used for which

$$
Y\left[\operatorname{tr}\left(\tau_{p, k}\right)\right]=1+\frac{\lambda_{k} \varepsilon}{\eta_{p, k}} \operatorname{tr}\left(\tau_{p, k}\right) .
$$

In the above expressions $\lambda_{k}$ stands for the relaxation time of mode $k, \eta_{p, k}$ is the polymer viscosity coefficient, $\mathbf{D}$ is the rate of deformation tensor, and $\varepsilon$ and $\xi$ are parameter coefficients of the PTT model that influence the extensional viscosity and the normal stress differences. Generally for each mode there is a set of model parameters, but some may be common to several modes, as convenient. In Eq. (5) the terms on the left-hand side are treated implicitly in the numerical solution of the corresponding algebraic equations, whereas those on the right-hand side go to the source term and are treated explicitly.

Results of numerical simulations are also presented for Newtonian fluids as well as for an inelastic fluid with a similar shear-thinning viscometric viscosity as the fitted four mode PTT model in order to assess separately the effects of shear thinning and fluid elasticity. 
The sets of governing equations [Eqs. (2)-(6)] are solved with the finite-volume method described in detail in our previous works [e.g., Oliveira et al. (1998); Alves et al. (2003a, 2003b)]. Basically, the solution domain is decomposed in a large number of adjacent control volumes over which those equations are volume integrated and transformed into algebraic form. These discretized matrix equations are then solved sequentially, for each dependent variable $\left(\mathbf{u}, p, \boldsymbol{\tau}_{p, k}\right)$, with conjugate gradient solvers. The meshes are nonstaggered and in order to ensure coupling between the velocity, pressure, and stress fields adequate formulations were developed for those quantities at the faces of the control volumes [Oliveira and Pinho (1999)] and a form of the SIMPLEC algorithm was adopted as described in detail in Oliveira et al. (1998).

Regarding the accuracy of the calculations, the discretization of the various diffusive terms of the governing equations was done by central differences, which is a secondorder scheme. For the convective terms, the CUBISTA high-resolution scheme of Alves et al. (2003a) was implemented in combination with the deferred correction approach of Khosla and Rubin (1974) to ensure stability and ease of implementation. The CUBISTA scheme has improved iterative convergence properties over classical high-resolution schemes, and has been applied and assessed in different types of flow solvers [e.g., Alves et al. (2003b); Santos et al. (2004); Carvalho et al. (2007); Ferreira et al. (2007)].

To deal with the multimode constitutive equation the modifications of the algorithm were minor. Instead of solving a single constitutive equation, the equations pertaining to the four PTT modes were sequentially solved at the step of the algorithm that deals with the solution of the constitutive model. Since in our methodology it is the iterative solution of the continuity equation that takes longer to converge, the computational time overload associated with the four modes is only incremented by about $20 \%$ relative to the single mode case.

\section{B. Constitutive equation}

Collis et al. (2005), among others, have pointed out to the need of employing multimode models in order to capture accurately the true relaxation response of actual polymer melts and solutions. Such recommendation has guided us in selecting the four-mode PTT constitutive model whose behavior has already been assessed in Sec. II for steady shear flow, by means of plots of the shear viscosity in Fig. 2 and the first normal stress difference coefficient in Fig. 3. However, the contraction flow also has important elongational flow regions and it is adequate at this stage to understand the behavior of the constitutive equation regarding its extensional viscosity. This is shown in Fig. 4 as a plot of both the steady extensional viscosity and the Trouton ratio versus the extensional rate of deformation in uniaxial extension. The behavior is typical of linear PTT models, with an increase in extensional viscosity to a plateau at high extensional rates, but the Trouton ratio keeps increasing to higher deformation rates because of the shear-thinning nature of the viscometric viscosity. For small values of $\varepsilon$ the plateaus of $\eta_{E}$ and $\operatorname{Tr}$ are inversely proportional to this parameter.

\section{Mesh characteristics}

Although the sudden contraction geometry is symmetric relative to the two middle planes, all simulations were carried out on meshes covering the full wall-to-wall geometry in both directions, thus allowing for prediction of possible nonsymmetric flow patterns that might arise [as in the work of Poole et al. (2007) for a cross-slot geometry]. As a consequence, the only boundary conditions needed were no-slip at the solid walls and prescribed inlet and outlet boundary conditions. The inlet and outlet planes were posi- 


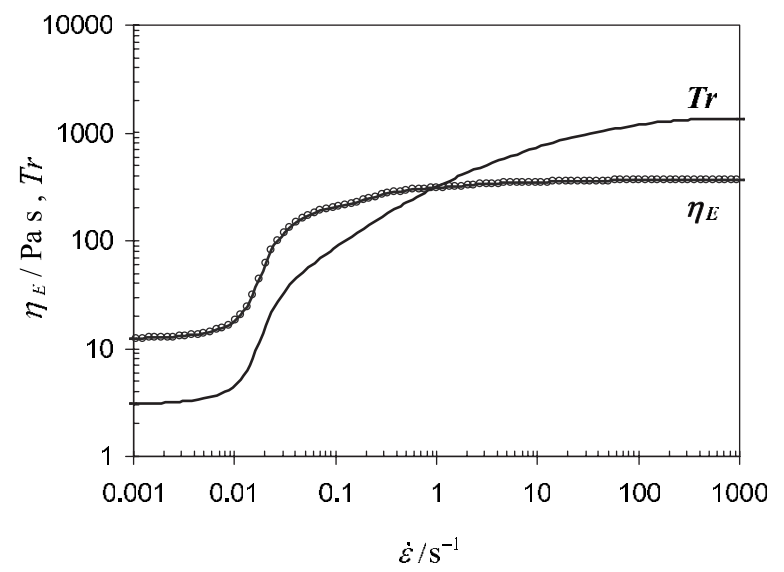

FIG. 4. Steady-state extensional viscosity and Trouton ratio for the four-mode PTT plus Newtonian solvent model (note that the shear viscosity used in the calculation of $\operatorname{Tr}$ is evaluated at $\dot{\gamma}=\sqrt{3} \dot{\varepsilon}$ ).

tioned very far from the region of interest $\left(-100 \mathrm{H}_{2}\right.$ and $100 \mathrm{H}_{2}$ for the Newtonian simulations-meshes M40 and M80;-166.7 $\mathrm{H}_{2}$ and $100 \mathrm{H}_{2}$ for the viscoelastic casesmeshes M40U and M64), so that fully developed flow conditions were enforced.

In the Newtonian calculations two meshes were used: mesh M40 had 40 cells in the upstream duct along each transverse direction, leading to a total of 51000 computational cells. The refined mesh M80 had twice as many cells in each direction so it had a total number of 408000 cells. The minimum normalized cell sizes $\left(\Delta x_{\min } / 2 \mathrm{H}_{2}=\Delta y_{\min } / 2 \mathrm{H}_{2}\right.$ $=\Delta z_{\min } / 2 H_{2}$ ) were 0.05 for mesh M40 and 0.025 for mesh M80. For the viscoelastic calculations two meshes were also used, one which had 40 uniform cells along each transverse direction (on the upstream channel), with a total of 126800 cells (mesh M40U) and another mesh with 56 nonuniform cells in each transverse direction (mesh M56), totaling 312816 cells. The minimum normalized cell sizes were 0.10 for mesh M40U and 0.03 for mesh M56, respectively. Note that all these meshes, although coarser than those in the detailed 2D study of Alves et al. (2003b), do represent a large increase in computational time because they correspond to $3 \mathrm{D}$ calculations leading to a significant increase in the number of degrees of freedom.

Local views of the four meshes in the contraction plane region are compared in Fig. 5 where the nonuniform mesh structure is clearly visible. Preliminary simulations were initially carried out with both meshes, for each fluid type, to ascertain mesh convergence. The results obtained with both grids are similar (to within $3 \%$ accuracy, or better) but to obtain accurate results we decided to carry out all the ensuing simulations with the refined meshes for each case (mesh M80 for the Newtonian simulations and mesh M56 with the viscoelastic fluid), unless otherwise mentioned.

\section{RESULTS AND DISCUSSION}

Comparison of experimental results from flow visualizations and numerical simulations are discussed first for Newtonian fluids and then for the viscoelastic fluid. Viscoelasticity induces the inversion of the recirculation patterns in the corner vortices formed upstream of the contraction, in addition to enhanced vortices, eventually leading to flow instability. 

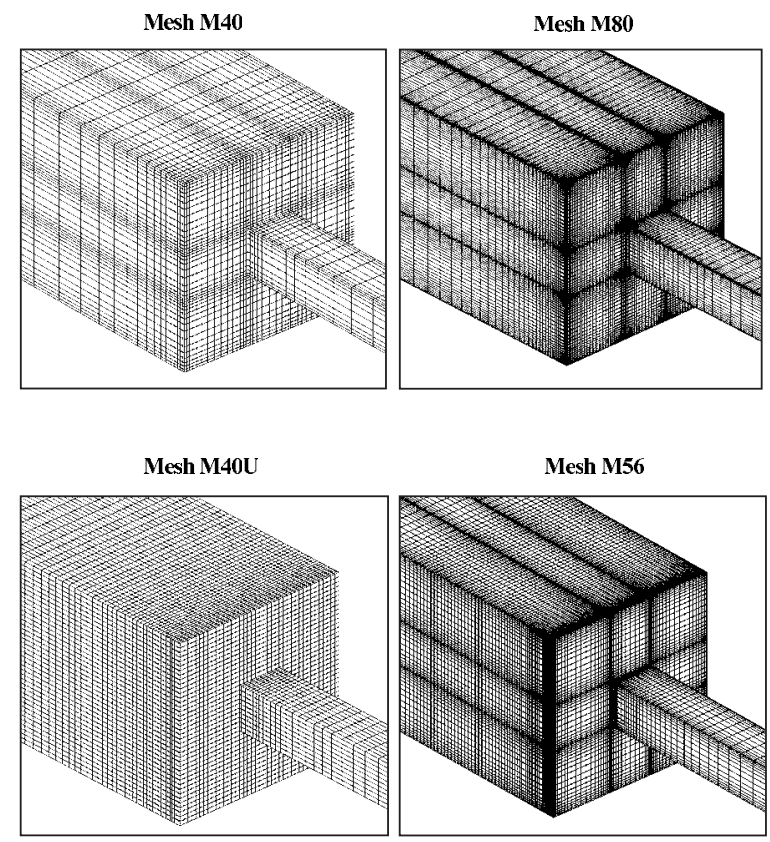

FIG. 5. Computational meshes M40, M80, M40U, and M56 in the vicinity of the contraction plane.

\section{A. Newtonian fluid flow}

Flow visualizations in the 4:1 SQ/SQ contraction at different Reynolds numbers were carried out first with Newtonian fluids and here comparisons were also made with results of numerical simulations, as shown in Fig. 6. The Reynolds number is defined on the basis of the downstream duct, as

$$
\operatorname{Re}_{2}=\frac{\rho U_{2}\left(2 H_{2}\right)}{\eta}
$$

All flow visualizations in this work represent stream traces in the middle plane $(z=0$ or $y=0$ planes) of the geometry. The numerical results shown on the right column of Fig. 6 reproduce well the visualizations: under conditions of negligible inertia the normalized vortex size obtained in the middle plane is $x_{R} / 2 H_{1}=0.163$ (cf. Fig. 6 for $x_{R}$ definition), but as inertia becomes non-negligible the size of the vortices decreases with Reynolds number and eventually the vortex vanishes at large flow rates. We note that due to symmetry the flow patterns are the same on the two middle planes $y=0$ and $z=0$. In Table III we present the predicted middle plane vortex sizes as function of the Reynolds number. These data have benchmark quality, although for the two highest Reynolds number flows there is still some mesh dependency. Assuming that the numerical method has second-order accuracy [cf. Alves et al. (2003a; 2003b)], we can predict the extrapolated (i.e., mesh independent) values of the vortex size, $x_{R \text {,ext }}$. The estimated error between the extrapolated values and the computed results on the refined mesh ranges from $0.1 \%$ for creeping flow, up to about $1.5 \%$ for the higher Reynolds number presented in Table III.

Even though the flow inside the vortices seen in Fig. 6 might look 2D, in reality it is highly $3 \mathrm{D}$ and none of the vortices are ever closed, in contrast with $2 \mathrm{D}$ flows. Under negligible inertia, the 3D flow pattern is rather complex and is illustrated in Fig. 7. Let us consider two different symmetry planes in Fig. 7: the middle (ABCD) plane perpendicu- 


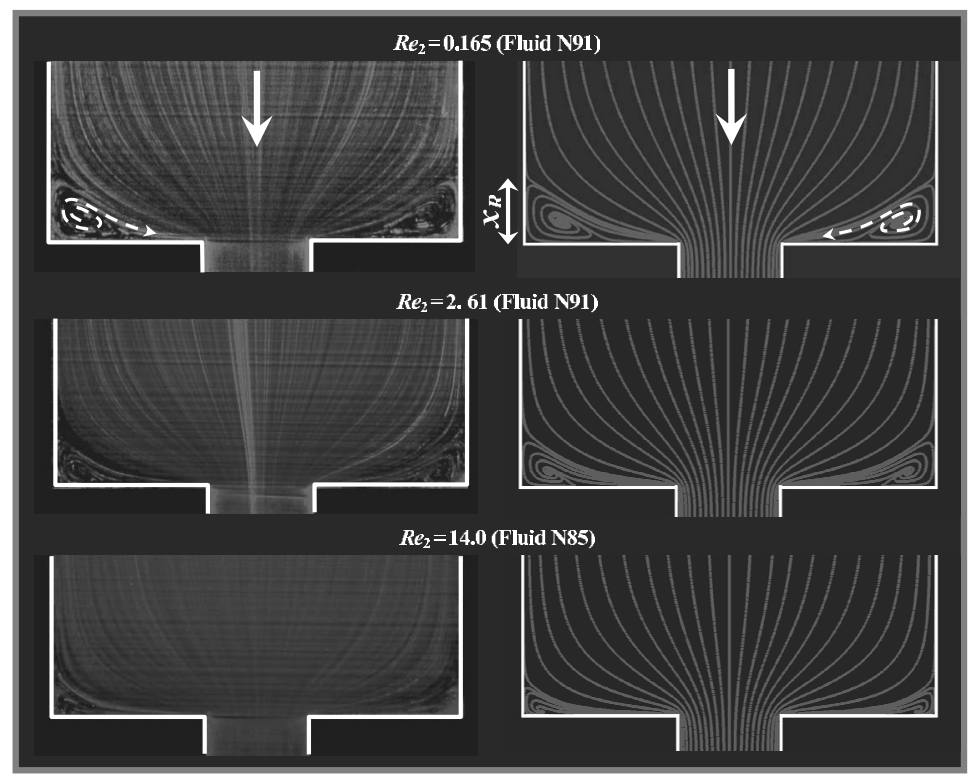

FIG. 6. Experimental (left column) and numerical (right column) streak lines for the flow of Newtonian fluids N85 and N91 in the middle plane of a 4:1 SQ/SQ sudden contraction.

lar to the wall and always represented in the photos; the second (EFGH) plane at $45^{\circ}$ to the wall and passing through opposite corners of the square cross section. In this second plane, henceforth referred to as corner (or diagonal) plane, no pictures could be taken but the descriptions below result from visual inspection of the flow as well as from the numerical calculations which were capable of capturing the observed flow features.

For the Newtonian fluids, the fluid particles entering the vortex at the corner plane, rotate towards its center and then drift along the eye of the 3D vortex towards the center of the middle-plane vortex. The particles at the middle-plane vortex rotate towards its periphery and exit the vortex at the reentrant corner flowing into the downstream duct, giving the impression that in this plane this vortex is closed. The experimental streak lines in the middle-plane vortex obtained by flow visualization, shown on the left side of Fig. 6, and the corresponding numerical streak lines, plotted on the right side, help understand the dynamics inside this vortex. In Fig. 7 we also present insets with the projected numerical streamlines on the middle and the diagonal planes, showing more clearly the different dynamics of the vortices on those two planes: while in the middleplane vortex the flow is spinning outwards, and leaving through the small channel, inside the corner-plane vortex the opposite is happening, i.e., the fluid coming from upstream near the intersection of the walls (along line HF) is captured by the corner vortex.

TABLE III. Influence of the Reynolds number on the vortex size in the middle plane for Newtonian fluid flow.

\begin{tabular}{cllll}
\hline \hline $\operatorname{Re}_{2}$ & 0 & 0.165 & 2.61 & 14.0 \\
\hline$x_{R} / 2 H_{1}$ (Mesh M40) & 0.1624 & 0.1597 & 0.1271 & 0.0756 \\
$x_{R} / 2 H_{1}$ (Mesh M80) & 0.1629 & 0.1602 & 0.1282 & 0.0792 \\
$x_{R, \text { ext }} / 2 H_{1}$ (Extrapolated) & 0.1631 & 0.1604 & 0.1286 & 0.0804 \\
\hline \hline
\end{tabular}




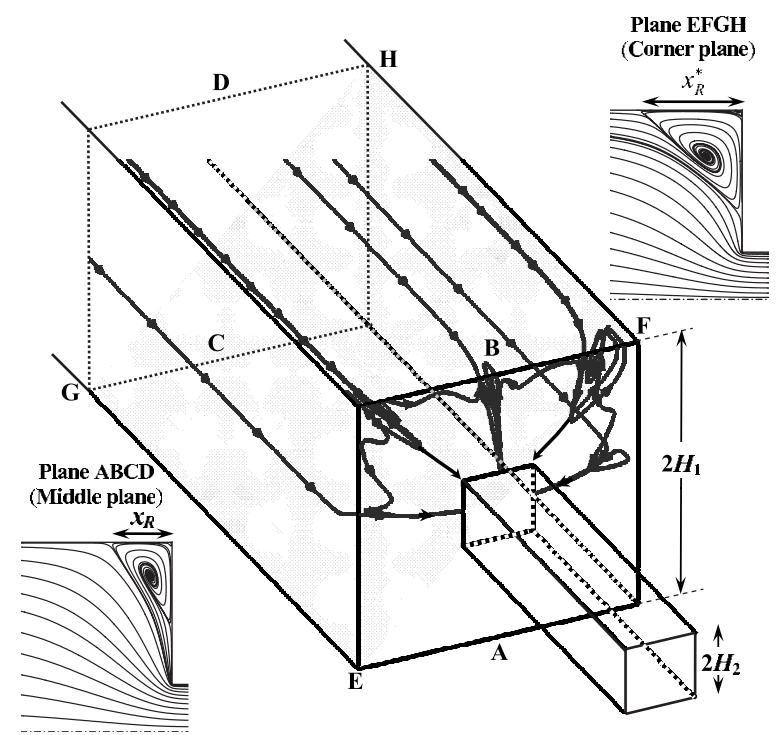

FIG. 7. Representative trajectories of fluid particles in the inertialess flow of a Newtonian fluid in the 3D 4:1 SQ/SQ sudden contraction. The projected stream traces on the middle and corner planes are also illustrated.

\section{B. Viscoelastic fluid flow}

The PAA500 fluid is shear thinning in viscosity, therefore the definition of Reynolds number is more ambiguous. Hence, the bulk velocity in the downstream duct is always indicated on the pictures. Note that the fluid has two limiting viscosities corresponding to the low and high shear-rate limits, which differ by a factor of 15 (cf. Fig. 2). In the definition of the Reynolds number [cf. Eq. (7)], we considered that the shear viscosity is evaluated at a characteristic shear rate of $\dot{\gamma}_{2}=U_{2} / H_{2}$.

To quantify the elasticity a Deborah number is defined in terms of downstream flow conditions,

$$
\mathrm{De}_{2}=\frac{\lambda_{M}(T) U_{2}}{H_{2}}=\frac{a_{T} \lambda_{M}\left(T_{0}\right) U_{2}}{H_{2}},
$$

where $\lambda_{M}$ represents Maxwell's relaxation time which is calculated from the linear viscoelastic spectrum according to

$$
\begin{gathered}
\eta_{p}=\sum_{k \neq \text { solvent }} \eta_{k}, \\
\lambda_{M}=\sum_{k \neq \text { solvent }} \frac{\eta_{k} \lambda_{k}}{\eta_{p}},
\end{gathered}
$$

thus, for the PAA500 fluid the following values are obtained: $\eta_{0}=\eta_{s}+\eta_{p}=4.07 \mathrm{~Pa} \mathrm{~s}, \beta$ $=\eta_{s} / \eta_{0}=0.0663$, and $\lambda_{M}=20.5 \mathrm{~s}$.

The influence of bulk velocity (or Deborah number), under conditions of low inertia, on the middle-plane vortex is illustrated by Fig. 8, which includes on the left column photos from the experimental visualizations, and on the right column streak lines from corresponding numerical simulations using the multimode PTT model. For this shearthinning fluid intense vortex enhancement is seen, as in the experimental observations of 



FIG. 8. Streak lines in the middle plane for the PAA500 fluid: experiments-left column; simulations-right column. (From top to bottom: $U_{2}=0.226,0.853,3.33,6.75 \mathrm{~mm} / \mathrm{s} ; \quad \mathrm{De}_{2}=1.54,5.82,22.7,46.1 ; \mathrm{Re}_{2}$ $=0.00051,0.0032,0.021,0.056$. 


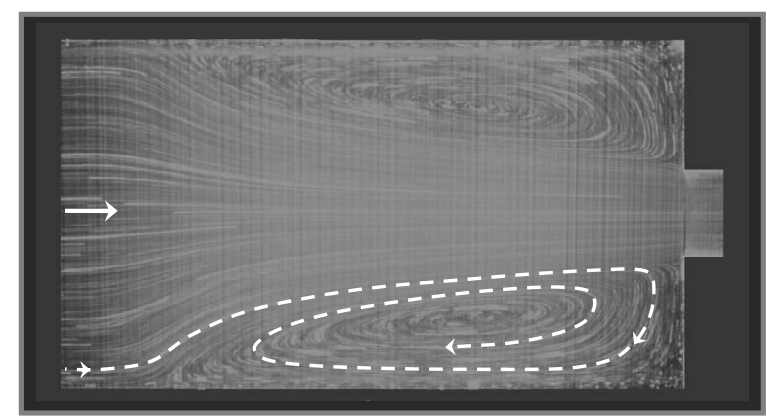

FIG. 9. Strong corner vortex enhancement for the flow of PAA500 fluid in the 3D 4:1 sudden contraction at $U_{2}=12.5 \mathrm{~mm} / \mathrm{s}\left(\mathrm{De}_{2}=85.3 ; \mathrm{Re}_{2}=0.128\right)$.

Evans and Walters (1986) for the axisymmetric contraction. In fact, elastic shear-thinning fluids typically exhibit an intense corner vortex growth both in planar and axisymmetric contractions. The comparison between these visualizations and the numerical streak lines are remarkably similar up to $U_{2}=6.75 \mathrm{~mm} / \mathrm{s}\left(\mathrm{De}_{2}=46.1\right)$. For higher flow rates values of $x_{R}$ are underpredicted, but further investigations are required here with different viscoelastic models that are able to better reproduce the rheological behavior of the PAA500 fluid, and more refined meshes. Nevertheless, we note that the calculations with mesh M56 are extremely expensive because of the very high relaxation time of the first mode: flow predictions at $U_{2}=6.75 \mathrm{~mm} / \mathrm{s}\left(\mathrm{De}_{2}=46.1\right)$ on this mesh took about 12 days of CPU time in a personal computer based on the processor AMD Athlon XP 2400+ with $1 \mathrm{GHz}$ of random access memory. Note that mesh M56 has 312816 computational cells, corresponding to 8758848 degrees of freedom, a value considerably higher than any work documented so far in the computational rheology literature.

A second difference relative to the Newtonian flow is the shape of the vortices: whereas the middle-plane Newtonian vortex had a clear concave shape, for the shearthinning fluid the vortex is neither concave nor convex at very low bulk velocities and becomes convex as the vortex grows with elasticity. The vortex length increases significantly with further increase in bulk velocity and at $U_{2}=12.5 \mathrm{~mm} / \mathrm{s}$, corresponding to a Deborah number of $\mathrm{De}_{2}=85.3$, the vortex is very large as seen in Fig. 9, with $x_{R} / 2 H_{1}$ $=1.47$. Further increase in the flow rate leads to a transition from the steady to a periodic unsteady flow and at $U_{2}=16 \mathrm{~mm} / \mathrm{s}$ the flow is already time dependent.

The vortex enhancement seen under steady flow conditions is much stronger for this shear-thinning fluid than was observed by Alves et al. (2005) for Boger fluids. With view of elucidating the role of elasticity, Fig. 10 shows the influence of the Deborah number on the vortex size measured on the center plane $\left(x_{R} / 2 H_{1}\right)$. This figure also includes the numerically predicted values of $x_{R} / 2 H_{1}$ for the PTT fluid, and the first point worth noticing is a sudden reduction in the numerical rate of increase of vortex length with bulk velocity at high Deborah number flows. However, up to $\mathrm{De}_{2} \approx 40$ the match between numerical predictions and experimental measurements is remarkable, in line with the agreement also shown in the comparisons of Fig. 8. We also include in Fig. 10 the vortex size on the corner plane $\left(x_{R}^{*} / 2 H_{1}\right.$ - cf. Fig. 7 for definition) predicted numerically, although for this plane no experimental data is available for comparison. Figure 10 also includes the predictions of $x_{R} / 2 H_{1}$ (and $x_{R}^{*} / 2 H_{1}$ ) for a generalized Newtonian fluid $(\mathrm{GNF})$, with a shear viscosity curve identical to that of the PAA500 fluid ( $\eta_{(\mathrm{GNF})}=0.27$ $+3.80 /\left[1+(20 \dot{\gamma})^{2}\right]^{0.25}$, with $\eta$ and $\dot{\gamma}$ expressed in Pa s and $\mathrm{s}^{-1}$, respectively). For this 


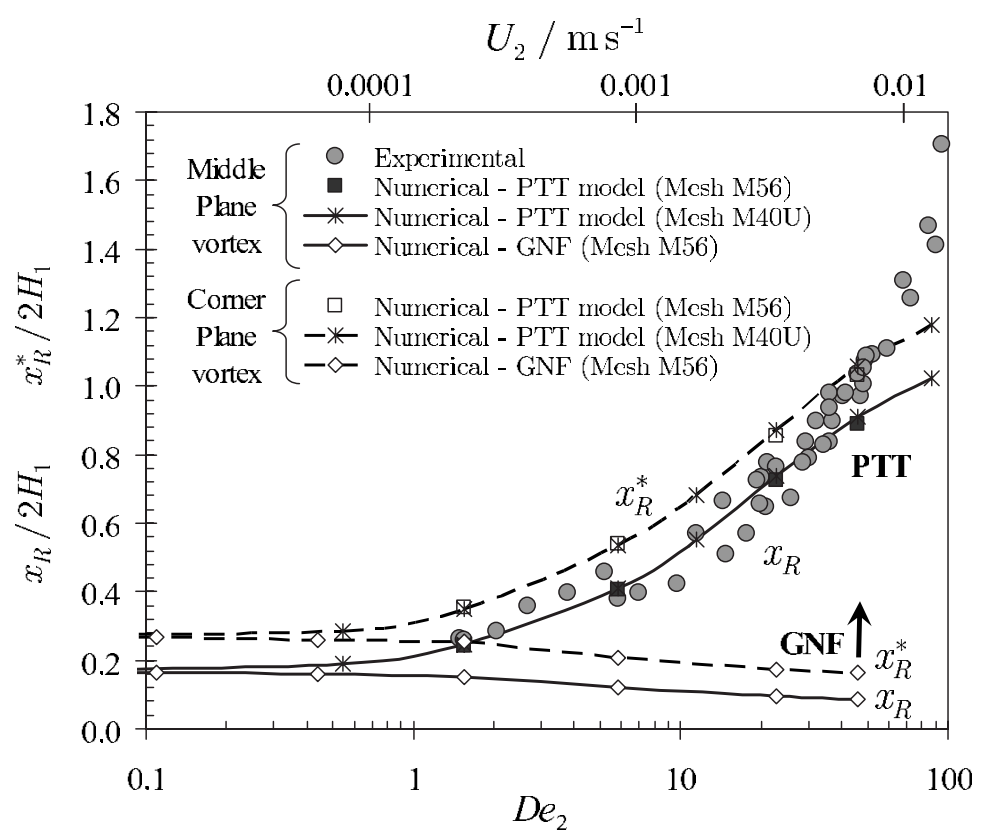

FIG. 10. Comparison between measured middle-plane vortex length in the SQ/SQ contraction for PAA500 fluid and predictions using a multimode PTT model. Also included are the predictions at the corner plane (dashed lines) and predictions obtained with a GNF with a shear-viscosity curve identical to that of the PAA500 fluid. In this case the results were obtained for the same flow rate conditions as the viscoelastic fluid.

inelastic fluid, the trend observed is not even qualitatively similar to the experimental data, thus demonstrating that the strong vortex enhancement observed is due to viscoelasticity, and more precisely as a result of the high extensional viscosity of the PAA500 fluid (cf. Fig. 4). In order to substantiate this explanation, we have conducted additional numerical simulations using different values of the $\varepsilon$ parameter of the PTT model and keeping the remaining parameters unchanged. Due to the unavailability of rheometric data for the PAA500 fluid in extensional flow, there is some ambiguity on the adequate selection of the $\varepsilon$ parameter, which influences primarily the extensional viscosity and to a much lesser extent the shear viscosity (and $\Psi_{1}$ ), as demonstrated in Fig. 11. Therefore, by varying this parameter we can assess how the extensional properties of the fluid influence the flow kinematics.

In Fig. 12 we plot the predicted vortex size as a function of $\mathrm{De}_{2}$ for different $\varepsilon$ values, illustrating that increasing the Trouton ratio (or the extensional viscosity) leads to a significant increase of the vortex size. This plot also confirms that in order to accurately predict the experiments a small $\varepsilon$ parameter should be selected, and the value $\varepsilon=0.02$ indicated in Sec. II B for the multimode PTT model is appropriate. At the lower $\varepsilon$ parameter simulated $(\varepsilon=0.01)$ numerical divergence was observed for $\mathrm{De}_{2}=46.1$. The rise in $x_{R}$ begins at $\mathrm{De}_{2} \approx 1$, that is $\dot{\gamma}_{2} \approx 0.05 \mathrm{~s}^{-1}$, which corresponds to a strain rate of $\dot{\varepsilon} \approx\left(U_{2, c}-U_{1, c}\right) / H_{1} \approx 2.1 U_{2}\left(1-1 / \mathrm{CR}^{2}\right) /\left(\mathrm{CR} \mathrm{H}_{2}\right) \approx 0.5 U_{2} / H_{2} \approx 0.025 \mathrm{~s}^{-1}$ (note that for a square channel the centerline velocity of a Newtonian fluid is nearly 2.1 times the average velocity in the channel: $\left.U_{2, c} / U_{2}=U_{1, c} / U_{1} \approx 2.1\right)$. From Fig. 11 it is confirmed that such value of $\dot{\varepsilon}$ correlates well with the initial sharp increase in the Trouton ratio. It is therefore established that the inception of vortex enhancement follows from a sudden rise in the Trouton ratio vs. $\dot{\varepsilon}$ curve for a given fluid. 


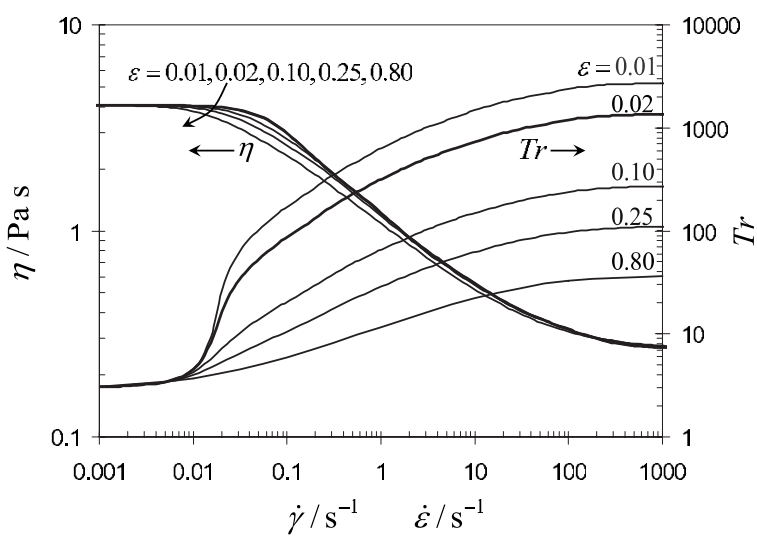

FIG. 11. Shear viscosity $\eta(\dot{\gamma})$ and Trouton ratio $\operatorname{Tr}(\dot{\varepsilon})$ for the four-mode PTT plus Newtonian solvent model with different $\varepsilon$ values (note that the shear viscosity used in the calculation of $\operatorname{Tr}$ is evaluated at $\dot{\gamma}=\sqrt{3} \dot{\varepsilon}$ ).

In Fig. 13 we plot predicted profiles of pressure along the centerline $(y=z=0)$, for a range of $\mathrm{De}_{2}$, for the parameters of the PAA500 fluid presented in Table II (with $\xi$ $=0.04$ and $\varepsilon=0.02$ ). In order to facilitate direct comparison between the different sets of data, we have normalized the pressure profiles as $\left(p-p_{\text {ref }}\right) /\left(2 \tau_{w, 2}\right)$, where the reference pressure, $p_{\text {ref }}$, was taken as the pressure at the location $x / H_{2}=-8$. The characteristic wall shear stress used in the normalization of the pressure profiles, $\tau_{w, 2}$, refers to the average value under fully developed flow conditions in the small channel, and can be related to the pressure gradient by $2 \tau_{w, 2}=H_{2}(-d p / d x)_{2, \mathrm{FD}}$, where $(d p / d x)_{2, \mathrm{FD}}$ represents the (con-



FIG. 12. Influence of the extensibility parameter $\varepsilon$ on the predictions of the middle-plane vortex size using the multimode PTT model (mesh M40U), and comparison with measured data in the middle plane of the SQ/SQ contraction for PAA500 fluid. Also included are the predictions with a generalized Newtonian model with a shear-viscosity curve identical to that of the PAA500 fluid. In this case the results were obtained for the same flow rate conditions as the viscoelastic fluid. 


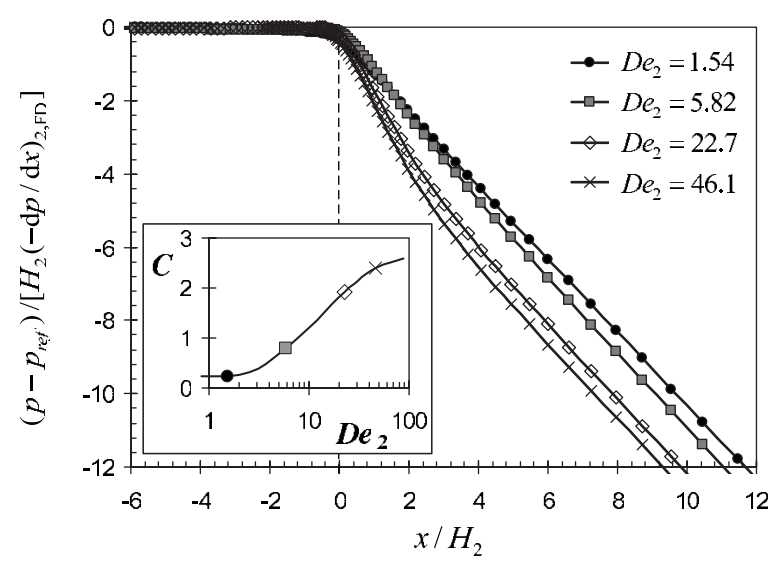

FIG. 13. Pressure profiles along the centerline for different Deborah numbers $\mathrm{De}_{2}$ predicted using the multimode PTT model on mesh M56. The inset shows the variation of the Couette correction with $\mathrm{De}_{2}$.

stant) pressure gradient on the downstream channel under fully developed flow conditions. As shown in Fig. 13, an increase of the flow rate (or $\mathrm{De}_{2}$ ) leads to a higher entrance pressure drop, which can be quantified using a Couette correction coefficient, $C$, as plotted in the inset. The Couette correction is defined as a normalized entry (or extra) pressure drop, $C=\left(\Delta p-\Delta p_{\mathrm{FD}}\right) / 2 \tau_{w, 2}$, where $\Delta p$ represents the total pressure drop between one location far upstream of the contraction plane, and another point located far downstream of the entry region. The extra pressure drop is given by $\Delta p_{\text {ext }}=\Delta p-\Delta p_{\mathrm{FD}}$, where $\Delta p_{\mathrm{FD}}$ represents the pressure drop that would be observed between those two locations if the flow was fully developed everywhere. The Couette correction is found to increase significantly with $\mathrm{De}_{2}$, and there is a correlation with the increase of the Trouton ratio observed as $\dot{\varepsilon}$ increases, as shown in Fig. 11. In addition, in Fig. 14(a) we analyze the influence of $\varepsilon$ parameter on the pressure profiles along the centerline, for a relatively large and constant flow rate $\left(U_{2}=6.75 \mathrm{~mm} / \mathrm{s} ; \mathrm{De}_{2}=46.1\right)$. It is clear that a decrease of $\varepsilon$ gives an enhancement of the entry pressure drop (as better illustrated in the Couette correction plot shown as inset), thus being demonstrated that higher extensional viscosities are directly related to larger flow resistance in the entrance region where extensional properties are all too important, as illustrated by the $N_{1}$ plots presented in Fig. 14(b). We note that even for the largest extensibility parameter tested $(\varepsilon=0.8)$ the behavior although approaching that of an inelastic fluid with a similar shear viscosity curve still shows signs of slight vortex enhancement, thus giving further evidence of the importance of polymer extensibility upon the rise in entry pressure drop and on vortex enhancement. It is important to emphasize here that although the shear viscosities are similar for the PTT model with a high $\varepsilon$ parameter (which is viscoelastic) and for the generalized Newtonian model (which is inelastic), in the latter there are no significant normal stresses in the extensional entry flow, while in the former those are not negligible as shown in Fig. 14(b), thus giving further credit to the importance of normal extensional stresses in the entry flow behavior.

Besides the enhanced corner vortex increase and the shape of the vortex, there is a third major difference between the viscoelastic and Newtonian fluid flow patterns: at high flow rates (or Deborah numbers) but still under conditions of steady flow, the direction of the secondary motion of fluid particles inside the vortices are inverted relative to those found at lower flow rates and with Newtonian fluids. This is clear from both the flow 

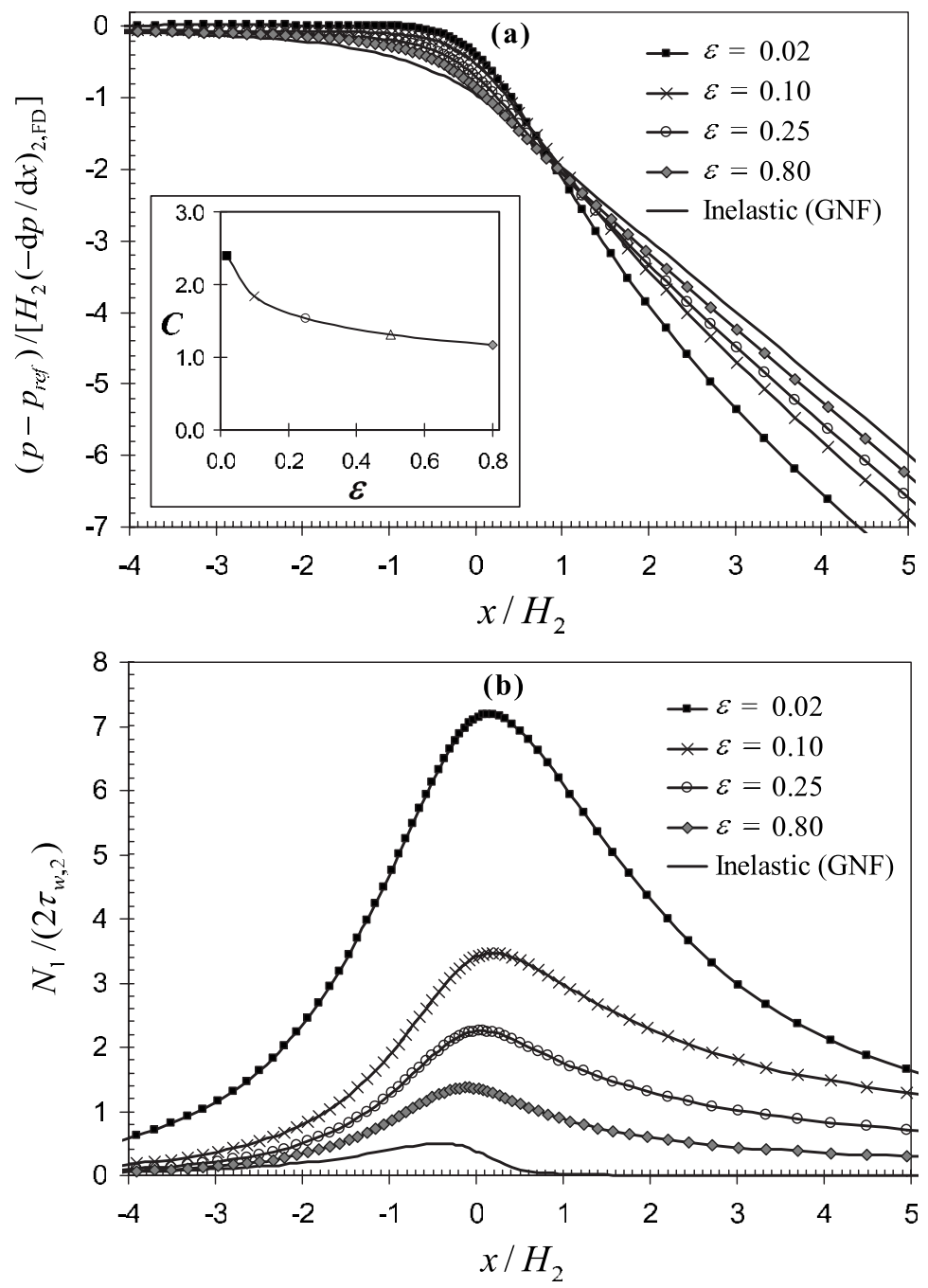

FIG. 14. Influence of the $\varepsilon$ parameter on the predicted (a) pressure and (b) $N_{1}$ axial profiles along the centerline, using the multimode PTT model (mesh M56) for $U_{2}=6.75 \mathrm{~mm} / \mathrm{s}\left(\mathrm{De}_{2}=46.1\right)$. Also included are the predictions with a generalized Newtonian model with a shear-viscosity identical to that of the PAA500 fluid and a plot of the Couette correction vs $\varepsilon$ [inset of (a)].

visualizations and the numerical simulations, in particular those at higher flow rates in Fig. 8. This new flow pattern is illustrated in the sketch of fluid particle trajectories in Fig. 15 that corresponds to a bulk velocity of $U_{2}=0.853 \mathrm{~mm} / \mathrm{s}\left(\mathrm{De}_{2}=5.82\right)$. In contrast to the observation for Newtonian fluids, the fluid coming from the upstream duct and entering the middle-plane vortex rotates towards its eye and then moves towards the eye of the corner-plane vortex where it rotates from the eye towards its periphery and exits the corner-plane vortex to the downstream duct. This flow pattern reversal is due to the effects of fluid elasticity and is well captured in the numerical simulations. The high extensional viscosity of the fluid is the most probable cause for this flow inversion since the effect is not captured by the simulations using the inelastic shear-thinning model under equivalent flow conditions. For all cases simulated numerically, we found that the flow inversion occurred when $x_{R} / 2 H_{1}>0.22 \pm 0.02$, thus indicating that vortex enhance- 


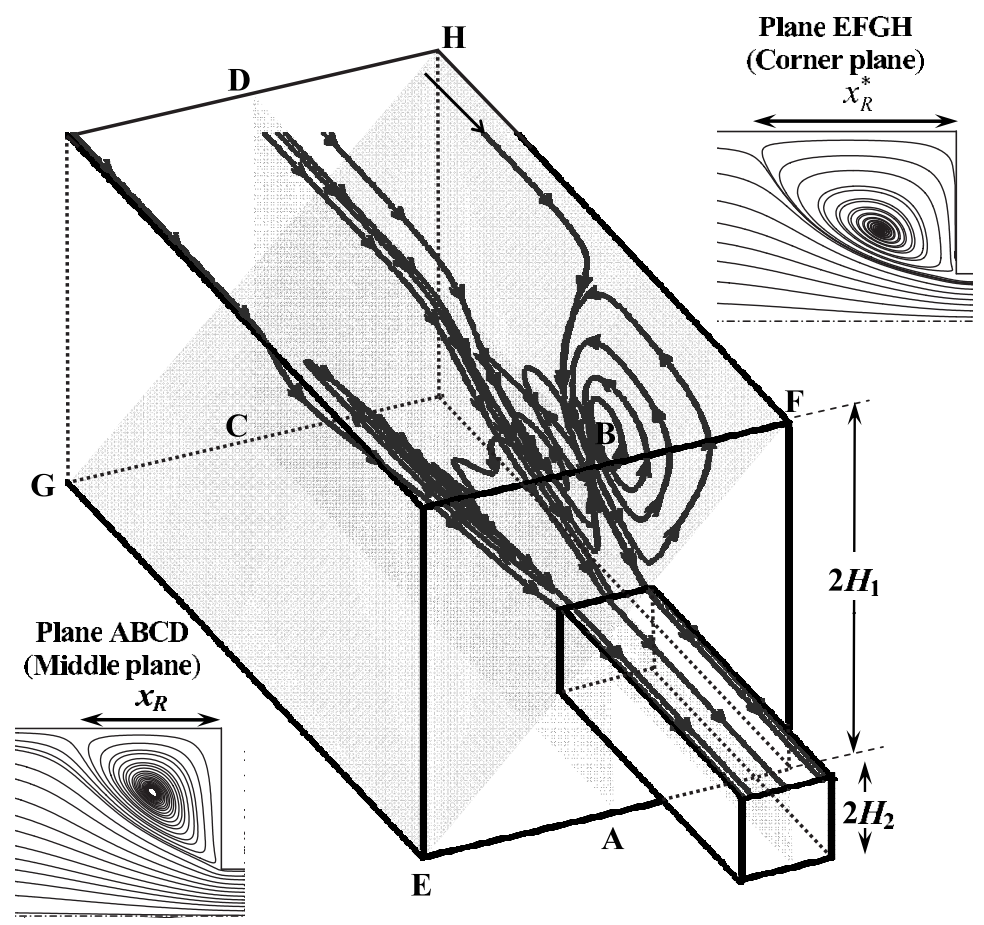

FIG. 15. Representative trajectories of fluid particles in the inertialess flow of PAA500 in the 3D 4:1 sudden contraction flow $\left(U_{2}=0.853 \mathrm{~mm} / \mathrm{s}, \mathrm{De}_{2}=5.82, \mathrm{Re}_{2}=0.0032\right)$. The projected stream traces on the middle and corner planes are also illustrated.

ment and flow inversion are related outcomes of fluid elasticity. For low values of $\varepsilon$, vortex enhancement is stronger and flow reversal occurs at lower values of $\mathrm{De}_{2}$. For $\varepsilon$ $=0.8$ flow reversal occurs for $\mathrm{De}_{2}$ between 22.7 and 46.1, while for $\varepsilon=0.02$ flow inversion is already observed at $\mathrm{De}_{2}=5.82$.

As already mentioned, at high flow rates the flow of PAA500 becomes unsteady but periodic, in the same way as previously observed for Boger fluids by Alves et al. (2005) in the same geometry. For an upstream bulk velocity of $U_{2}=18.2 \mathrm{~mm} / \mathrm{s}$, which corresponds to $\mathrm{De}_{2}=124$ and $\mathrm{Re}_{2}=0.208$, the sequence of photographs presented in Fig. 16 represents three different moments within a cycle of flow periodicity. Obviously, the periodicity is happening in both transverse directions. From films taken with a movie camera at a known frame rate it was possible to estimate the frequency $(f)$ of oscillation for different flow rates within the periodic regime. This frequency varied linearly with flow rate defining a constant Strouhal number $\mathrm{St}=2 f H_{1} / U_{1} \approx 0.5$.

We speculate that at higher flow rates, not attained in these experiments, further elastic instabilities will grow and turn the flow chaotic. Predictions of the observed flow features will require a full $3 \mathrm{D}$, time-dependent simulation of the whole geometry with an adequate constitutive model, a challenge to be undertaken in the future.

\section{CONCLUSIONS}

Flow visualizations and numerical simulations were carried out in a 4:1 sudden SQ/SQ contraction for Newtonian and viscoelastic shear-thinning fluids under conditions of low 




FIG. 16. Instantaneous flow patterns for a supercritical flow rate for fluid PAA500 $\left(U_{2}=18.2 \mathrm{~mm} / \mathrm{s}, \mathrm{De}_{2}\right.$ $=124, \operatorname{Re}_{2}=0.208$ ).

inertia. For the 3D numerical simulations a finite-volume code was used and a four-mode linear PTT plus Newtonian solvent model simulated the rheology of the non-Newtonian fluid.

For the Newtonian fluid the numerical flow patterns in the middle plane are in excellent agreement with experimental results. The flow field is clearly 3D with fluid particles moving from the upstream duct and entering the corner-plane vortex, rotating towards its center, and then drifting to the eye of the middle-plane vortex where they rotate to the outside of the vortex and exit at the reentrant corner into the downstream duct. For the non-Newtonian fluid the visualizations reveal that very significant changes in fluid dynamics are taking place under inertialess flow conditions: the vortices became convex in shape and grew significantly in size due to fluid elasticity, and the vortices and the dynamics of the secondary flow at high flow rates have been reversed with fluid particles entering the middle-plane vortex and exiting at the corner-plane vortex. At even higher flow rates the long vortices became unstable and a periodic flow emerged. The numerical simulations predicted well the measured flow patterns at low flow rates provided the extensibility parameter of the PTT model was properly chosen, but underpredicted the vortex length at higher flow rates near the critical conditions that lead to the onset of an elastic instability. No simulations were attempted under these critical conditions where unsteady flow is observed. It was demonstrated that the elongational properties of the fluid are a key quantity in determining correctly both the size of the vortices formed upstream of the contraction and the entry pressure drop. 


\section{ACKNOWLEDGMENTS}

M.A.A. acknowledges funding by Fundação para a Ciência e a Tecnologia (Portugal) and FEDER through program POCI 2010 (project POCI/EQU/59256/2004).

\section{References}

Alves, M. A., P. J. Oliveira, and F. T. Pinho, "A convergent and universally bounded interpolation scheme for the treatment of advection," Int. J. Numer. Methods Fluids 41, 47-75 (2003a).

Alves, M. A., P. J. Oliveira, and F. T. Pinho, "Benchmark solutions for the flow of Oldroyd-B and PTT fluids in planar contractions," J. Non-Newtonian Fluid Mech. 110, 45-75 (2003b).

Alves, M. A., D. Torres, M. P. Gonçalves, P. J. Oliveira, and F. T. Pinho, "Visualization studies of viscoelastic flow in a 4:1 square/square contraction," COBEM 2003, 17th International Congress of Mechanical Engineering, São Paulo, Brazil 2003, 2003c.

Alves, M. A., P. J. Oliveira, and F. T. Pinho, "On the effect of contraction ratio in viscoelastic flow through abrupt contractions," J. Non-Newtonian Fluid Mech. 122, 117-130 (2004).

Alves, M. A., F. T. Pinho, and P. J. Oliveira, "Visualizations of Boger fluid flows in a 4:1 square-square contraction," AIChE J. 51, 2908-2922 (2005).

Alves, M. A., and R. J. Poole, "Divergent flow in contractions," J. Non-Newtonian Fluid Mech. 144, 140-148 (2007)

Boger, D. V., "Viscoelastic flows through contractions," Annu. Rev. Fluid Mech. 19, 157-182 (1987).

Boger, D. V., and K. Walters, Rheological Phenomena in Focus (Elsevier, Amsterdam, 1993).

Boger, D. V., D. U. Hur, and R. J. Binnington, "Further observations of elastic effects in tubular entry flows," J. Non-Newtonian Fluid Mech. 20, 31-49 (1986).

Brown, R. A., and G. H. McKinley, "Report on the VIIIth International Workshop on Numerical-Methods in Viscoelastic Flows,” J. Non-Newtonian Fluid Mech. 52, 407-413 (1994).

Cable, P. J., and D. V. Boger, "A comprehensive experimental investigation of tubular entry flow of viscoelastic fluids: Part I. Vortex characteristics in stable flow,” AIChE J. 24, 868-879 (1978a).

Cable, P. J., and D. V. Boger, "A comprehensive experimental investigation of tubular entry flow of viscoelastic fluids: Part II. The velocity fields in stable flow,” AIChE J. 24, 992-999 (1978b).

Cable, P. J., and D. V. Boger, "A comprehensive experimental investigation of tubular entry flow of viscoelastic fluids: Part III. Unstable flow,” AIChE J. 25, 152-159 (1979).

Carvalho, J. R. F. G., J. M. P. Q. Delgado, and M. A. Alves, "Diffusion cloud around and downstream of active sphere immersed in granular bed through which fluid flows," Chem. Eng. Sci. 62, 2813-2820 (2007).

Coates, P. J., R. C. Armstrong, and R. A. Brown, "Calculation of steady-state viscoelastic flow through axisymmetrical contractions with the EEME formulation," J. Non-Newtonian Fluid Mech. 42, 141-188 (1992).

Collis, M. W., A. K. Lee, M. R. Mackley, R. S. Graham, D. J. Groves, A. E. Likhtman, T. M. Nicholson, O. G. Harlen, T. C. B. McLeish, L. R. Hutchings, C. M. Fernyhough, and R. N. Young, "Constriction flows of monodisperse linear entangled polymers: Multiscale modeling and flow visualization," J. Rheol. 49, 501522 (2005).

Crochet, M. J., A. R. Davies, and K. Walters, Numerical Simulation of Non-Newtonian Flow (Elsevier, New York, 1984).

Evans, R. E., and K. Walters, "Flow characteristics associated with abrupt changes in geometry in the case of highly elastic liquids," J. Non-Newtonian Fluid Mech. 20, 11-29 (1986).

Evans, R. E., and K. Walters, "Further remarks on the lip-vortex mechanism of vortex enhancement in planar contraction flows," J. Non-Newtonian Fluid Mech. 32, 95-105 (1988).

Ferreira, V. G., C. M. Oishi, F. A. Kurokawa, M. K. Kaibara, J. A. Cuminato, A. Castelo, N. Mangiavacchi, M. F. Tomé, and S. McKee, "A combination of implicit and adaptative upwind tools for the numerical solution of incompressible free surface flows," Commun. Numer. Methods Eng. 23, 419-445 (2007). 
Hassager, O., "Working group on numerical techniques. Fifth International Workshop on Numerical Methods in Non-Newtonian Flows, Lake Arrowhead, USA," J. Non-Newtonian Fluid Mech. 29, 2-5 (1988).

Khosla, P. K., and S. G. Rubin, "A diagonally dominant second-order accurate implicit scheme," Comput. Fluids 2, 207-209 (1974).

Lee, K., M. R. Mackley, T. C. B. McLeish, T. M. Nicholson, and O. G. Harlen, "Experimental observation and numerical simulation of transient stress fangs within flowing molten polyethylene," J. Rheol. 45, 12611277 (2001).

McKinley, G. H., W. P. Raiford, R. A. Brown, and R. C. Armstrong, "Nonlinear dynamics of viscoelastic flow in axisymmetric abrupt contractions," J. Fluid Mech. 223, 411-456 (1991).

Mompean, G., and M. Deville, "Unsteady finite volume simulation of Oldroyd-B fluid through a threedimensional planar contraction,” J. Non-Newtonian Fluid Mech. 72, 253-279 (1997).

Oliveira, P. J., and F. T. Pinho, "Numerical procedure for the computation of fluid flow with arbitrary stressstrain relationships," Numer. Heat Transfer, Part B 35, 295-315 (1999).

Oliveira, P. J., F. T. Pinho, and G. A. Pinto, "Numerical simulation of non-linear elastic flows with a general collocated finite-volume method," J. Non-Newtonian Fluid Mech. 79, 1-43 (1998).

Oliveira, M. S. N., F. T. Pinho, P. J. Oliveira, and M. A. Alves, "Effect of contraction ratio upon viscoelastic flow in contractions: The axisymmetric case," J. Non-Newtonian Fluid Mech. 147, 92-108 (2007).

Owens, R. G., and T. N. Phillips, Computational Rheology (Imperial College Press, London, 2002).

Phan-Thien, N., and R. I. Tanner, "A new constitutive equation derived from network theory," J. NonNewtonian Fluid Mech. 2, 353-365 (1977).

Poole, R. J., M. A. Alves, and P. J. Oliveira, "Purely-elastic flow asymmetries," Phys. Rev. Lett. 99, 164503 (2007).

Purnode, B., and M. J. Crochet, "Flows of polymer solutions through contractions. Part 1: Flows of polyacrylamide solutions through planar contractions," J. Non-Newtonian Fluid Mech. 65, 269-289 (1996).

Quinzani, L. M., R. C. Armstrong, and R. A. Brown, "Birefringence and laser-Doppler velocimetry (LDV) studies of viscoelastic flow through a planar contraction," J. Non-Newtonian Fluid Mech. 52, 1-36 (1994).

Rothstein, J. P., and G. H. McKinley, "Extensional flow of a polystyrene Boger fluid through a 4:1:4 axisymmetric contraction/expansion,” J. Non-Newtonian Fluid Mech. 86, 61-88 (1999).

Santos, J. C., P. Cruz, M. A. Alves, P. J. Oliveira, F. D. Magalhães, and A. Mendes, "Adaptive multiresolution approach for two-dimensional PDEs,” Comput. Methods Appl. Mech. Eng. 193, 405-425 (2004).

Sirakov, I., A. Ainser, M. Haouche, and J. Guillet, "Three-dimensional numerical simulation of viscoelastic contraction flows using the Pom-Pom differential constitutive model," J. Non-Newtonian Fluid Mech. 126, 163-173 (2005).

Walters, K., and D. M. Rawlinson, "On some contraction flows for Boger fluids," Rheol. Acta 21, 547-552 (1982).

Walters, K., and M. F. Webster, "On dominating elastico-viscous response in some complex flows," Philos. Trans. R. Soc. London, Ser. A 308, 199-218 (1982).

Walters, K., and M. F. Webster, "The distinctive CFD challenges of computational rheology," Int. J. Numer. Methods Fluids 43, 577-596 (2003).

Xue, S. C., N. Phan-Thien, and R. I. Tanner, "Three dimensional numerical simulations of viscoelastic flows through planar contractions,” J. Non-Newtonian Fluid Mech. 74, 195-245 (1998a).

Xue, S. C., N. Phan-Thien, and R. I. Tanner, "Numerical investigations of Lagrangian unsteady extensional flows of viscoelastic fluids in 3-D rectangular ducts with sudden contractions," Rheol. Acta 37, 158-169 (1998b) 